\title{
Efficient Content Discovery in P2P-Multimedia System
}

\author{
V. Kavitha Chandrakanthan \\ Senior Assistant Professor \\ Dept. of Computer Science \& Engineering \\ Christ College of Engineering \& Technology \\ Pondicherry University
}

\author{
R. Raj Priyadarshini \\ M. Tech Final Year \\ Dept. of Computer Science \& Engineering \\ Christ College of Engineering \& Technology \\ Pondicherry University
}

\begin{abstract}
In this paper, we are interested to provide fast content discovery in P2P multimedia systems. To constructs AVL tree for best efficient search peer in P2P multimedia system. Gossip protocol to exchange information to its neighbouring peers and which chunks to be downloaded from which peer. An issue in peer management manages the scheduling algorithm from which peer. The analysis is made on hybrid content discovery in P2P multimedia system and how the chunk should be distributed over the peer and also how the request chunk picking from the peers in structured networks to be focused and issues are stated. The comparative study is based on the existing features to be undertaken with structure and unstructured networks for fast and efficient content discovery in P2P Multimedia system.
\end{abstract}

Keywords: P2P Multimedia, Gossip protocol, Content discovery.

\section{INTRODUCTION}

Peer-to-Peer (P2P) systems have become popular recent technology to provide multimedia content. P2P Multimedia system has an ability of sharing resources among the peers. Well-organized and fast content discovery algorithm is significant to Peer-to-Peer multimedia system. Hence, it reducing buffering delay after seek operation is performed. In order to exchange information in a computer network have been followed rules and data formats in Communication protocols which provide on the basic for network programming. Peer-to-Peer multimedia system may includes the mixture of still, video, text, audio and interactivity content forms. When compared with centralized networks, P2P networks each peer can act as client and server at the same time for same operations.

For Example, Uploading/downloading the video file leads to simple and efficient content to be discover in P2P Multimedia system. To search the video content in structured search network efficiently using gossip protocol.

Peer-to-Peer multimedia system supports user interactivity such as video cassette Recorder (VCR), the first popular technology to provide users with time control over their video. VCR operations will make enormous shock on data dissemination. Peer accepts the video-on-demand service, it should store the media data onto its own storage systems and its neighbours while one requesting the peer. Video server is located in the CCRNC (Center China Regional Network Center) of CERNET (China Education and Research Network). In P2P networks which include bandwidth, storage space and computing power while clients provide resources.
In $\mathrm{P} 2 \mathrm{P}$ networks increases robustness in decentralized nature, because it removes the single point failure in centralized server. Since, May 2006 Gridcast has been prepared and deployed on China's CERNET. In binary balanced tree structure in which each node of the tree is maintained by a peer. In AVL tree each node can hold several video file according to the request of user the root node can create a holder chain. After receiving video file then return back to the corresponding peer [1]. P2P overlay network logic which is used to organize the data managed by multiple self-directed computers. Topologies have been suggested for this network including a ring topology [2].

In Peer-to-Peer multimedia system content of video segmentation to be divided the video into multiple pieces. To locate a specific chunk in Distributed Hash Table (DHT) by using VMesh. Peer-to-Peer system Gossip method in an unstructured overlay to discover only chunk.

Here, peer can exchange the data availability information to its neighbors periodically [3]. P2P overlay network properties are:

- Self - organizing

- Efficient use of resources

- Scalability

- Reliability

- Ease of deployment and administration.

In this paper, we conduct an in-depth study of P2P Multimedia based on a real-world P2P Multimedia system built and deployed in Hybrid overlay network for efficient search the Video content in structured search networks. AVL tree is a load balancing which will reduces the searching delay in P2P Multimedia systems.

The rest of this paper is Structured as follows Section 2 we discuss the Related work, Section 3 we present the Basic Design, Section 4 we present the classification of overlay Networks, Section 5 presents the comparison on unstructured and unstructured overlay P2P network Section 6 Concludes the paper.

\section{RELATED WORKS}

Information source in video is commonly used for capturing and sharing resources in P2P multimedia Systems. In P2P Multimedia Systems is difficult to accessing the resources in unstructured tree. From this point of view, P2P Multimedia systems provide watch when downloading and uploaded the file user experience. Kangaroo utilizes centralized chaser may leads to single point of failure while searching the video files and it becomes a bottleneck of the system [4]. The gossip protocol which could discover the content in P2P-multimedia system it make use of storage forwarding schemes. In Buffer forwarding schemes, the P2P Multimedia can stored a video content recently played at particular period before closing the application. In order to get a video file from RAM. In storage 
forwarding schemes, the P2P Multimedia can stored a video content in local storage devices (i.e.) Hard disk. While enclosing the application the content must be static. In P2P caching a technique used to reduces bandwidth cost for content on P2P networks. In P2P Multimedia content discovery the distributed Hash table (DHT) the key and value pair allocate for each and every nodes in search network. While the user entry the key value based on key the node in AVL tree to be searched.

When a peer wants to start watching a movie, it goes to the tracker to find out the movies from which peer have that movie part. The neighboring peer can also handled the same video files and other part of the movie files. Based on the structure search network the part of video files from various peer can get back according to the user request. Fast and capable content to be discover efficiently in P2P Multimedia system.

In the beginning, the Distributed Hash Table(DHT) are a class of decentralized distributed system that offer a lookup Service comparable to hash table(Key, Value) pair are stored in the DHT and any participating peer can efficiently retrieve the value associated with a given key value. DHT-Based networks have been widely utilized for accomplishing efficient resource discovery for grid computing system. In Decentralized resource discovery have been based on extending the existing DHTs with the capability of multidimensional data organization.

The work is differ from existing work because in this paper integrate a structured overlay with gossip protocol to discover the content from one peer to its neighbouring peers. The gossip protocol exchange the information from one peer to its neighbouring peer without any delay make use of storage forwarding systems. The video or music files exchanged by BitTorrent as illegal files. The video data is distributed over a chain format in a peer. The groups of peer forward the stream within the group or between groups. The plan of bartering for chunks of a file from BitTorrent [5] which is very admired $\mathrm{P} 2 \mathrm{P}$ system for off-line downloading the video using the Give-To-Get Algorithm. Once the peers have been downloaded the full video in structure network, there is enough upload bandwidth to maintain free-riders without compromising the well-behaving peers. [6]

\section{BASIC DESIGN}

This section describes the basic design of Grid cast in details and how the Gridcast works for peer sharing the resources in terms of peer management and also describes distribution and chunk picking.

\subsection{Peer Management}

All of the peers are watching the same video into an overlay network organized by Gridcast. Generally, there are three key issues in peer management.

- Sharing the peer concerts between each other in P2P overlay network.

- Peer selection leads to data sharing between each peer in structure overlay network make much more efficient and best peer.

- Using Gossip protocol spread the information to each peer in the P2P network.

Finally request for scheduling, it can determine from which peer the chunk will be downloaded. The user requested the data may be distributed over the peer (i.e.) different system.
Since, it is distributed application of Peer-To-Peer multimedia network system.

Manage the peer in structured search network the video files can be divided into separate chunk stored in AVL tree. Based on the key value in Distributed Hash Table the value should be search from top of the root node to the leaf node either may left child or right child. Without any delay searching the file efficiently in search network based on storage forwarding schemes.

\subsection{Chunk Distribution}

In chunk distribution peers forward chunk of a video file to each other. The full video file/data is split up into chunks of equal size. When the user request for the video files, the peer retrieving every video data to be appends from the each peer in hybrid networks.

In P2P network, spread the information to each peer based on structured search network using gossip protocol for information exchange from various peer the chunk must be distributed over it.

\subsection{Chunk Picking}

A Peer request the chunk from other peer. A peer can decide in which order it will request the chunks to be downloaded the chunk is called chunk picking. While many number of peer perform the same operation (i.e.) requesting the same video file. While picking the chunk to playback the video files/data then seek operation to be performed. Based on the priority, the operation to be undertaken to picking the chunk whenever Upload/Download the video file in P2P networks.

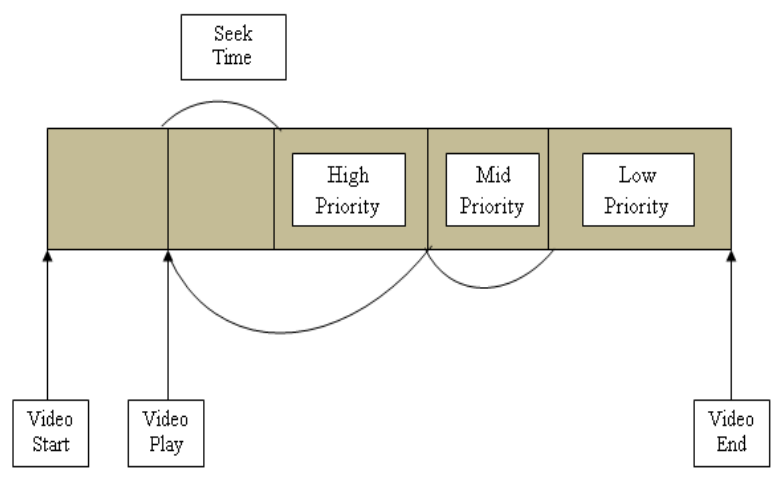

Fig 1: Priority based chunk picking

\section{CLASSIFICATION OF OVERLAY NETWORKS}

In this section, we present the general classification of overlay networks in $\mathrm{P} 2 \mathrm{P}$ system are structured and unstructured overlay network.

\subsection{Structured overlay networks}

AVL tree is used to solve searching problems most frequently. The client search from the root node can achieve best efficiency search in structure networks. It can create a holder chain to carry out the chunks which are available in P2P network. Holder chain can hold all the available chunk in AVL tree.AVL is implemented in distributed systems, tree nodes are distributed across network. The Topology construction is based on Node ID's that are generated by using Distributed Hash Tables (DHT).In the Structured overlay 
network assigns keys to each data items in the P2P Multimedia network. The network is based on storing the Video files/objects in structured overlay network. It Guarantees object detection in $\mathrm{O}(\log \mathrm{n})$ hops from the content Addressable Networks (LAN), chord.

\subsection{Unstructured overlay networks}

In unstructured overlay networks self-possessed of peers joining the network with some loose rules, without any prior knowledge of the topology. Networks have been followed random walks to send query across the overlay with the limited scope. When a peer receives the several query, it sends a list of all context matching the query (video file given by user) to the originating peer (i.e.) Server. Unstructured P2P File sharing Network are:

\subsubsection{Centralized directory based P2P systems}

In Centralized server, all the peer are connected to central entity (central server). Each and every Peer establishes connection between each other video on demand when video is available to exchange the information to the corresponding user.

\subsubsection{Pure P2P systems}

Without any centralized server, all the peer connected to any terminal entity. If any of the peers has been removed means without loss of functionality left over then peers are working condition. User could able to get the video file from any of the peers. Pure P2P systems reusable for future use.

\subsubsection{Hybrid P2P systems}

Hybrid P2P systems is the combination of star, ring, mesh topology. In Hybrid context discovery mechanism follows the distributed application (Distributed server). Each leaf nodes connected to one or more super peers. In Distributed P2P system participating peer as network node can acts as server of client. Distributed sever working at any condition without loss of operational in peers. Getting back of video file from any one of the neighbouring peers even any one of the peer had been loss of video files.

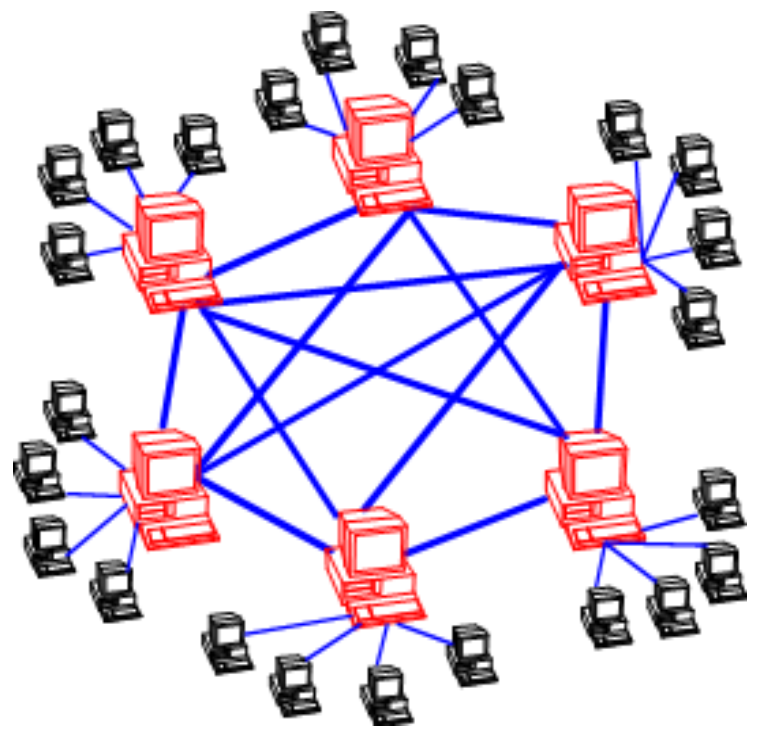

Fig 2: Hybrid P2P Multimedia System

\section{COMPARISON ON OVERLAY P2P NETWORKS}

This section describes the basic comparison made on unstructured and structure overlay $\mathrm{P} 2 \mathrm{P}$ networks for improving the searching time efficiently in $\mathrm{P} 2 \mathrm{P}$ Multimedia system using structured search network.

Table 1. Comparison table for overlay P2P Networks

\begin{tabular}{|c|c|c|c|}
\hline S. No & Contents & Unstructured & Structure \\
\hline 1 & Searching speed & Leads to high & Very low \\
\hline 2 & Replication & $\begin{array}{c}\text { High replicating } \\
\text { the data }\end{array}$ & $\begin{array}{c}\text { Minimum } \\
\text { Replication }\end{array}$ \\
\hline 4 & Traffic & Heavy Traffic & No Traffic \\
\hline 5 & Dree search & Difficulty & Simplicity \\
\hline
\end{tabular}

This section describes the basic comparison made on unstructured and structure overlay $\mathrm{P} 2 \mathrm{P}$ networks for improving the searching time efficiently in $\mathrm{P} 2 \mathrm{P}$ multimedia system using structured search network.

\section{CONCLUSION AND FUTURE WORK}

In this paper, we have presented a novel to reduce search delay in P2P Multimedia system the analysis made on efficient search based on structure network. The peer exchange information from one peer to its neighbouring peer. Peer management shows that how chunk distribution have been take place and to picking the chunk from the peer. Various schemes in structured and unstructured P2P overlay networks that have been classified. The comparative study is undergone with different structured and unstructured networks. In future, we plan to introduces a Internet Routing protocol for checking bandwidth using Gossip like exchange information in P2P Multimedia system.

\section{REFERENCES}

[1] H. V. Jagadish, B. C. Ooi, and Q. H. Vu, "BATON: A balanced tree structure for peer-to-peer networks," in Proc. 31st Int. Conf. Very Large Data Bases (VLDB '05), 2005, pp. 661-672.

[2] I.Stoica, R. Morris, D. Karger, M. F. Kaashoek, and H. Balakrishnan,"Chord: A scalable peer-to-peer lookup service for internet applications," SIGCOMM Comput. Commun. Rev., vol. 31, no. 4, pp. 149-160, 2001.

[3] Y. Huang, T. Z. Fu, D.-M. Chiu, J. C. Lui, and C. Huang, "Challenges, design and analysis of a large-scale P2P VoD system," in Proc. ACM SIGCOMM 2008 Conf. Data Communication (SIGCOMM '08), New York, 2008, pp. 375-388. 
[4] X. Yang, M. Gjoka, P. Chhabra, and P. Rodriguez, "Kangaroo: video seeking in P2P systems," in Proc. $8^{\text {th }}$ Int. workshop Peer-To-Peer systems(IPTPS'09),2009

[5] Zhou Zhiqiang, Noriaki Yoshiura, "Detection of the Music or Video Files in BitTorrent", Theory and Practice of Computation Proceedings in Information and Communications Technology, Vol 5, pp 202-213, 2012.

[6] J.J.D.Mol, J.A.Pouwelse, M.Meulpolder, D.H.J.Epema, and H.J.Sips, "Give-To-Get: Free-riding-resilient videoon-demand in P2P systems".

[7] Y. He and Y. Liu, "VOVO: VCR-oriented Video-onDemand in largescale peer-to-peer networks," IEEE Trans. Parallel Distrib. Syst., vol.20, pp. 528-539, Apr. 2009.

[8] Y. Guo, S. Yu, H. Liu, S. Mathur, and K. Ramaswamy, "Supporting VCR operation in a mesh-based P2P VoD system," in Consumer Communications and Networking Conference, 2008. CCNC 2008. $5^{\text {th }}$ IEEE, Jan. 2008, pp. 452-457.

[9] Gongwei Fu, Hailong Sun, "Complexity Analysis of Random Search with AVL Tree" April 17, 2006.

[10] J.J.D. Mol, J.A. Pouwelse, M. Meulpolder, D.H.J. Epema, and H.J. Sips "Give-to-Get: An Algorithm for P2P Video-on-Demand" Delft University of Technology, The Netherlands.

[11] N. Magharei and R. Rejaie, "PRIME: Peer-to-Peer ReceiverdrIven MEshed-Based Streaming System," Proc. IEEE INFOCOM,2007.
[12] Knuth, J.H. Morris, and V. Pratt, "Fast Pattern Matching in Strings," SIAM J. Computing, vol. 6, pp. 323-350, 1977.

[13] K. Sripanidkulchai, A. Ganjam, B. Maggs, and H. Zhang, "The Feasibility of Supporting Large-Scale Live Streaming Applications with Dynamic Application EndPoints," Proc. ACM SIGCOMM,2004.

[14] M. Conti and S. Giordano, "Multihop ad hoc networking: the theory," Communications Magazine, IEEE, vol. 45, pp. 78-86, April 2007.

\section{AUTHORS PROFILE}

Kavitha received the B.E. degree under Department Computer Science and Engineering in 2002 affiliated to Madras University and the M.E. degree in Computer Science and Engineering in 2007 affiliated to Anna University. She is currently working as a Senior Assistant Professor in the Department of Computer Science and Engineering from Christ College of Engineering and Technology, Pondicherry.

Raj Priyadarshini received the B.E. degree under Department Computer Science and Engineering from Mailam Engineering College in 2011 affiliated to Anna University. She is currently pursuing the M. Tech degree in the Department of Computer Science and Engineering from Christ College of Engineering and Technology affiliated to Pondicherry University 